\title{
Autonomous Control System for an Electric ATV
}

\author{
Cosmin Rus ${ }^{1, *}$, Monica Leba ${ }^{1}$, Nicoleta $\mathrm{Negru}^{2}$, Răzvan Marcuş² and Alin Costandoiu ${ }^{2}$ \\ ${ }^{1}$ University of Petrosani, Department of Automation, Computers, Electrical Engineering and \\ Energetics, University Street 20, Petroșani, 332006, Romania \\ ${ }^{2}$ Doctoral School of University of Petrosani, University Street 20, Petroșani, 332006, Romania
}

\begin{abstract}
This paper lays the foundations of an autonomous control system for an electric ATV type vehicle. The conversion of a classic motor vehicle into an electric one is shortly presented and the main advantages deriving from such a motorization especially in terms of the main impact on the environment in the sense that the noxious substances resulting from a classic thermal motorization are eliminated and the use of the vehicle can be extended for industrial halls too. This electric vehicle has, in addition to the classic proximity sensors, a high-resolution LIDAR type scanning system that allows it to map an enclosure so that the processed data can then be used in the autonomous driving algorithm. The LIDAR type scanning system is also correlated with a location system based on a LoRa communication system to allow a predictive location of the route followed by the autonomous vehicle. It is desired to use the whole complex, especially in some industrial halls and in the current context it is suitable to be used as a disinfection vehicle in order to prevent the personnel health issues in the context of SARS-COV2 epidemics.
\end{abstract}

\section{Introduction}

Autonomous vehicles are a life-saving technology and, if improved, could significantly reduce the number of deaths caused by driver errors while driving on public roads. The technology used by these vehicles to measure and interpret the world around them in real time works in several ways using several specific algorithms. Today's autonomous vehicles are largely based on an established technology, known as light detection and measurement, or LiDAR. According to many specialized works in the field as well as many reports, road accidents cause 1.3 million deaths worldwide each year, and according to US regulatory statistics, $94 \%$ of them are attributed exclusively to human error. As presented in the fieldspecific literature only between 2014 and 2016, the number of vehicle deaths increased by $14 \%$ in the United States, an increase that some analysts believe is due to increased mobile phone use and distracted driving [1].

An autonomous vehicle is a vehicle that can interact with the environment on its own, by this meaning other vehicles, traffic signs, roads or pedestrians, and drive safely without the driver having to intervene in the entire driving process. This car does not need the

\footnotetext{
*Corresponding author: cosminrus@upet.ro
} 
presence of a human passenger to take control and is capable of anything a car with a driver can do [2]. Autonomous vehicles are based on a wide range of sensors and actuators and in terms of software on complex algorithms and dedicated processors to run the programs behind the optimal operation. These vehicles create and maintain environmental maps based on a variety of sensors located in various parts of the car. Radar sensors monitor the positions of nearby cars. Video cameras detect traffic lights, read traffic signs, monitor other vehicles and pedestrians. LIDAR sensors (light detection) bounce light from objects around the car to measure distances, to detect road edges and markings. Ultrasonic sensors detect curves and other vehicles when parking. Sophisticated software processes all this data, creates a route and sends instructions to the actuators that control acceleration, braking and steering. Various software applications or libraries, reliable obstacle avoidance algorithms, predictive modelling of road behaviour and recognition of surrounding objects help the software to track traffic lights and navigate safely [3]. Currently, there are 5 levels of automation, from 0 (full manual) to 5 (full autonomous) [4].

Level 0 - full manual: The driver is $100 \%$ responsible for the operation of the vehicle. However, the system can send alerts to warn the driver of dangers.

Level 1 - driving assistance: An automatic driving system assists the driver in the driving process. The system can manoeuvre the steering wheel or control the speed, but it cannot do both at the same time.

Level 2 - partial automation: A human driver and the automatic system share the responsibility of driving the car. The system can turn the steering wheel and control the speed of the car simultaneously, while the driver monitors the system.

Level 3 - conditioned automation: The automatic system can drive the car and monitor the environment. Drivers are free to take their eyes off the road, but must remain in the driver's seat in case they need to intervene.

Level 4 - high automation: The system can drive the car, monitor the environment, and does not require human intervention. However, at this level, the system is limited in special situations, such as traffic congestion.

Level 5 - full autonomous: The car is $100 \%$ autonomous, does not need human intervention and can drive on all roads and in various environmental conditions.

In Europe, vehicles with automation levels 3 and 4 are expected to enter the market by 2030 and because of this there are still not enough data to make an analysis on all aspects of the industry. Instead, vehicles that assist the driver in the driving process (vehicles with automation level 1 and 2) are already a common presence on public roads in Europe. According to specialized studies conducted in Europe (Deloitte Automotive Consumer) it is shown that European citizens are still very sceptical about autonomous vehicles.

Autonomous cars offer many advantages in terms of quality of life: they can help the elderly and the disabled to have more independence, you can send objects to other localities without having to travel, or you can send your dog to the vet if you are too busy to take him, for example. Autonomous cars offer more free time. On average, a person can spend between one and two hours a day driving. When the autonomous car takes care of everything that means driving, you can relax in the back seat, reading a book or watching a movie. In addition to these daily benefits, autonomous cars also have global benefits: they reduce carbon dioxide emissions, reduce traffic congestion, reduce transportation costs, free up parking spaces [5]. An important advantage is to increase safety by reducing the number of victims of road accidents. Like all good things, autonomous cars have disadvantages. One of them would be the loss of many jobs. The ultimate goal of autonomous cars is to relieve the entire responsibility of driving a car in the currently known sense. When $100 \%$ autonomous cars are sold on a large scale, people who rely on driving to earn a living will lose their income. People who manage an entire fleet of drivers will have to fire employees. The same can happen with companies that offer services such as driving lessons or taxi. 
The existence of autonomous cars also raises questions related to the moral part. Regarding the development of autonomous vehicles, there are several paradigms that must be discussed and analysed, especially from an ethical point of view. The first question is: are autonomous cars capable of making quick decisions and making moral judgments at the same level as people? If a person jumps in front of an autonomous car, and the car has to choose between hitting the person or turning to the sidewalk, what will the car choose? The second major question that arises: if the car is really capable of making moral decisions, is it okay to allow it? A third major disadvantage of autonomous cars is the possibility of being stolen by hacking. Car thieves generally use physical objects to get into the vehicle, but autonomous cars can also become vulnerable if the thief's re-profile themselves on the computer hacking side. Paradoxically, as cars have become more sophisticated, their computer systems offer hackers more vulnerabilities. A cyberattack on an autonomous car can lead to traffic jams or fatalities from accidents. Autonomous cars also have some other limitations and dilemmas that manufacturers are trying to solve. One of them would be the fact that the LIDAR sensor is extremely expensive and is still trying to find the perfect balance between distance and resolution. If several autonomous cars would drive on the same road, the question arises whether their LIDAR sensors will interfere. Another limitation is related to the weather. For example, what happens if the snow covers the markings on the road? Or if the road is covered with water, debris or oil stains? Will autonomous cars be able to run smoothly in tunnels or bridges? How will they manage in bar-to-bar traffic conditions? Who is responsible in the event of an accident: the manufacturer, the human passenger? The latest research shows that level 5 autonomous cars will not even be equipped with a steering wheel, so the passenger will not be able to take control in case of an emergency. Autonomous cars seem to be the cars of the future, but it remains to be seen how far away this future is. It is clear that they have many advantages, but until all the dilemmas and limitations listed above are solved, their mass production is still impossible [6].

Many papers are currently dealing with the algorithms needed for a vehicle to become autonomous and there are more and more unusual approaches that all have the role of providing reliable algorithms especially for the rest of the traffic participants [7].

\section{Purpose of the study}

The design and construction of an autonomous vehicle aims to replace the people in the driving loop and ensure a safe travel environment. The autonomous vehicle must be able to understand the environment, estimate its location (road signs, static and dynamic obstacles, pedestrians) and make driving decisions based on known factors [8]. An autonomous vehicle observes its world through different sensors and has different communication protocols that allow it to integrate into the ecosystem of a smart city. The autonomous vehicle collects the data from sensors taking into account the possibilities of errors using instruments based on probability and thus creates a model of the environment in which it moves. Several techniques are available to determine important features of an autonomous vehicle such as position and speed. All methods test possible scenarios and then choose the best one that represents the conditions of the vehicle. After the initial condition of the autonomous vehicle has been accurately estimated, the next challenges are to plan the movement along a given trajectory and to control the system to ensure the correct movement. Motion planning is performed to determine the optimal route of a vehicle moving from an initial position to an imposed position. The command-and-control algorithms are designed to ensure movement from start to finish, with high energy efficiency and special robustness. Cruise control found in many of today's standard vehicles 
is one such algorithm that has been developed to maintain a constant speed. Today, Tesla autonomous cars are among the most popular on the market and at the same time are among the most technologically developed. The autonomous operation of these vehicles depends on a combination of global maps of the surroundings and reactive responses to nearby identified objects using a lot of specific sensors. However, the storage capacity of Tesla car batteries and therefore the increase in the number of kilometres that can be travelled on a single charge has been the main reason why these vehicles are popular all over the world although the acquisition costs are relatively high [9]. The problem to be solved by this paper lies in the need to create a hardware and software structure that allows the use of a vehicle as an autonomous vehicle in order to use it in an interior space to measure various environmental parameters or in the current epidemiological context, for disinfecting some spaces.

\subsection{Mobile platform development}

The research on modelling, designing and making an autonomous vehicle had as a starting point the conversion of an ATV (All-Terrain Vehicle) with a classic thermal engine (2cylinder gasoline engine $250 \mathrm{~cm}^{3}$ ) in an electric vehicle [10]. The heat engine part and the command-and-control part of this engine were removed and the functional platform of this vehicle was prepared so that an electric motor could be mounted. The chosen electric motor is one of the $2.2 \mathrm{~kW}$ three-phase asynchronous motor type, 1420 rotations / minute and $\cos \varphi$ 0.82 , protection class IP 44 . The power supply of this motor is made with the help of a $12 \mathrm{~V}$ battery, $240 \mathrm{Ah}, 1200 \mathrm{~A}$. The battery is connected to the circuit through a voltage inverter $12 \mathrm{~V}-220 \mathrm{~V}$ of $3000 \mathrm{~W}$. This inverter has the role of allowing the power supply of a Yaskawa VS-606V7 frequency converter with a power of $4 \mathrm{~kW}$. The three-phase motor is controlled from the three-phase output of the frequency converter. Figure 1 shows the electric vehicle obtained from the conversion of the vehicle with a classic heat engine.

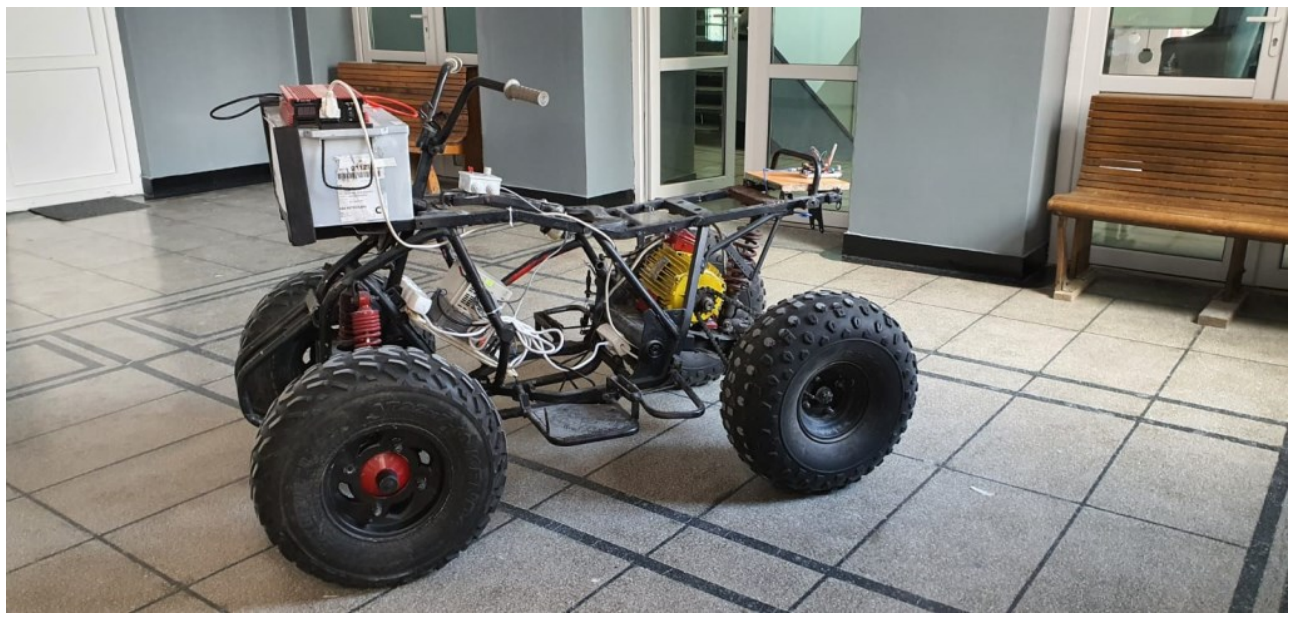

Fig.1. Electric vehicle (electric ATV)

The control part of the motor is made analogically using the control and command interface of the frequency converter by means of a potentiometer with which the motor speed is adjusted and by means of two contacts with which the operating direction can be selected (forward - reverse) [11]. 


\subsection{Lidar based mapping}

LiDAR is an electronic system that is part of the family of extremely sensitive sensors but very simple to use, more precisely in the category of Time to Flight (ToF) sensors. Most of the sensors used in the development of current autonomous vehicles gather concrete data about a specific physical parameter, such as temperature level, humidity level, light radiation level, while a LiDAR system measures in real time the distance to the nearest obstacle through a specific protocol and provides the vehicle with extremely accurate data about its position in the reference space. Lidar equipment is an acronym for "light detection and distance" and is the equivalent of radar for the visible light field. It is a method of measuring distances by illuminating the target, in this case an obstacle appeared in the path of the electric vehicle, with laser light and measuring the reflection using a sensor [12]. The calculation formula to calculate the distance to an obstacle using a LiDAR is:

$$
\text { Distance }=(\text { Speed of Light } x \text { Time of Flight }) / 2
$$

Among the advantages of a LiDAR are: Data can be collected quickly and accurately; LiDAR can be integrated with other sensors: sonar sensors, camera, IMU, GPS, ToF; thanks to an active lighting sensor, LiDAR technology can be used throughout the day (24 hours a day); It can be used to collect data from hard-to-reach places; LiDAR's are fast and extremely accurate. These sensors are excellent tools for collecting data on large areas of land; Once properly configured, a LiDAR is a stand-alone technological piece and can work quite a lot without intervention on it.

Disadvantages of LiDAR are: LiDAR can be expensive depending on the specifications required by the project; LiDAR's are ineffective in heavy rain, thick clouds, if there is heavy fog or smoke or transparent obstacles are used; analysing the massive amount of data collected can consume time and resources; powerful laser blades used in some LiDAR's can damage the human eye; difficulties in penetrating extremely dense matter.

According to all the research studied, it can be said that LiDAR sensors are a vital technology in the process of making a vehicle that can achieve the highest degree of autonomy. Using LiDAR systems together with video cameras and radar sensors, autonomous vehicles perceive the environment, detect obstacles in a short time and thus ensure increased safety and efficiency on public roads [13].

The $360^{\circ}$ RPLIDAR A2 laser scanner developed by SLAMTEC has an extremely reliable and highly accurate laser measuring system and has excellent performance in indoor and outdoor work environments, but it is recommended not to be exposed to direct sunlight for those better performance (fig. 2). It can measure up to 4,000 laser samples per second.

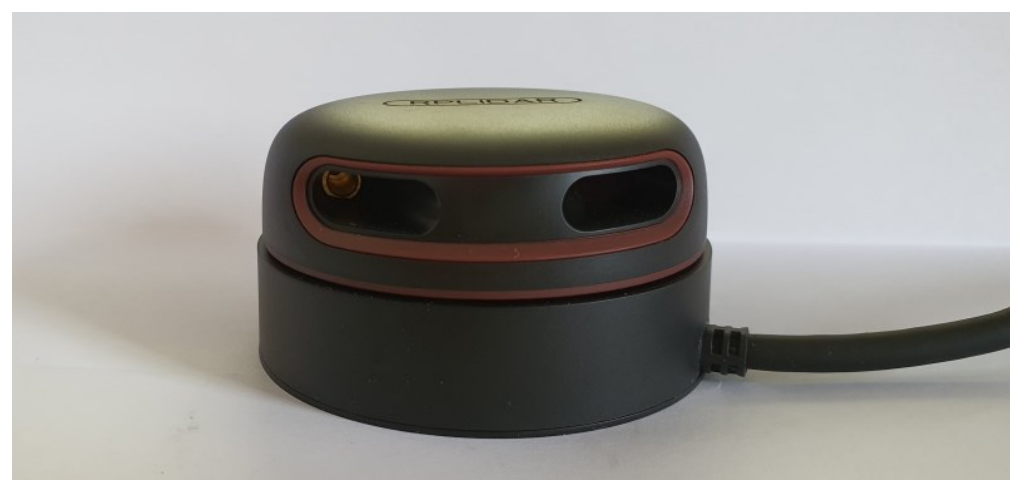

Fig. 2. RPLidar A2 $360^{\circ}$ 
According to the catalogue sheet the system can perform a 360-degree 2D scan within 6 meters. The generated data can be used in mapping, locating and modelling object / environment [14]. An example of use is shown in figure 3. Using an SDK utility in the Windows operating environment, it was possible to generate the map of the LA1 laboratory from the University of Petrosani, university where all the tests were performed.

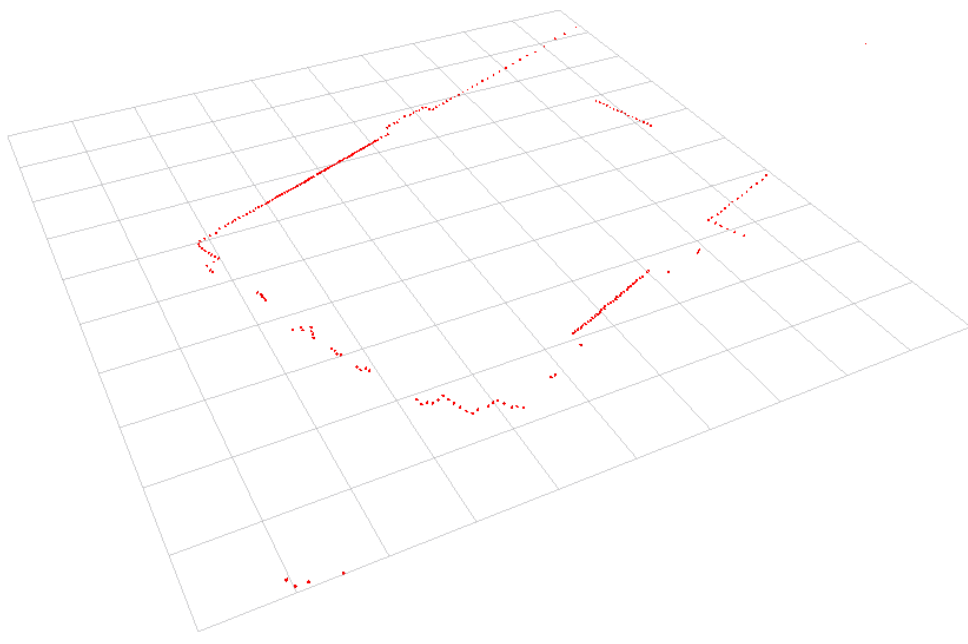

Fig. 3. Generating a map in Windows using RPLiDAR

Following the tests performed, it was found that the typical scanning frequency of the RPLIDAR A2 equipment is $10 \mathrm{~Hz}$ (which represents $600 \mathrm{rpm}$ ). Under these conditions, the effective resolution of the scanning system will be $0.9^{\circ}$. The scan frequency can be adjusted in the range of $5 \mathrm{~Hz}$ to $15 \mathrm{~Hz}$ depending on the application requirements.

\subsection{LoRa based localization}

LoRa is a technology that provides smart solutions for developers with long-range, secure data transmission with low power consumption. Public and private networks that use this technology can provide coverage that is greater than that of existing cellular networks. It is an easy technology to connect to any existing infrastructure. LoRa uses the unlicensed subgigahertz $868 \mathrm{MHz}$ radio frequency band in Europe. LoRaWAN is the network on which LoRa operates. LoRaWAN is a radio transmission protocol that forms networks of intelligent objects, with Media Access Control (MAC) level and defines the communication protocol and system architecture for an Internet of Things network, while LoRa physical components allow the communication link over long distances.

LoRaWAN is perfect for endpoints with wireless communication and battery power from an IoT network, being able to provide secure two-way communications at different data transfer speeds. Typical applications of LoRaWAN are sensors networks, security systems, smart homes, smart metering devices, industrial control and smart cities [15].

The range of $2-5 \mathrm{~km}$ in urban areas and up to $15 \mathrm{~km}$ outside is possible through a balance between the range of communication and the duration of the message over a broad spectrum at sub-GHz frequencies, and an adaptive speed scheme of data transfer. 
LoRaWAN operates in star topology, where the gates provide a transparent bridge that sends and receives messages between end devices and a central network or cloud.

Depending on the application chosen, the final devices may have different classes of functionality, as follows:

- Class A - The class of final devices of the lowest power, where the devices can transmit (uplink) whenever they want, but the reception windows (downlink) are allocated only after the transmission.

- Class B - The network provides a beacon signal that devices use to open reception windows at scheduled times, when they can transmit.

- Class C - The most energy-intensive mode of operation, Class C devices being able to receive continuously, unless they transmit.

Gates, typically power devices, communicate via the LoRaWAN protocol with endpoints before transmitting messages through traditional protocols such as Ethernet, 3G or Wi-Fi. Most endpoints have two-way communication, but in any case, LoRaWAN supports multiple broadcasts for node upgrade or mass distribution of emergency messages. Four LoRa devices were used in this research, each with the possibility of being either a node or a base station [16].

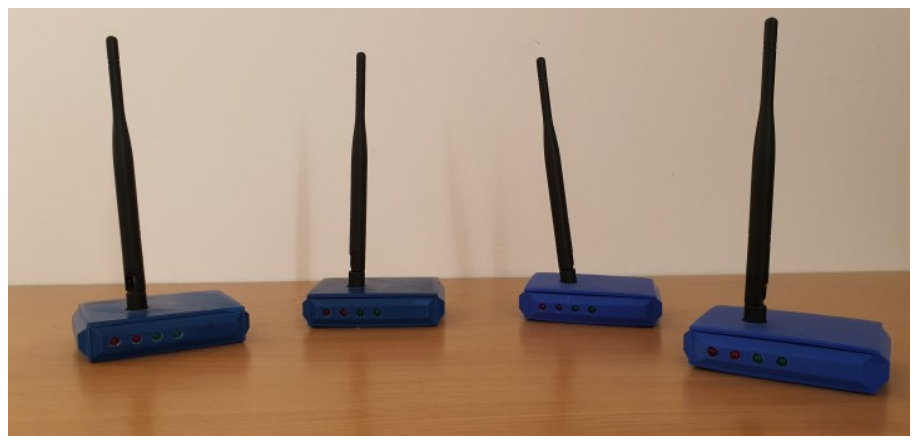

Fig. 4. The 4 LoRa modules used in the experiment

LoRa devices (fig. 4) were manufactured using ESP8266 and LoRa RFM96W transceiver modules (fig. 5). ESP8266 is a chip system (SoC) that integrates a 32-bit microcontroller, standard digital peripheral interfaces, RF antenna, power amplifier, low noise reception amplifier, filters and allows module power management in a small package. RFM96W transmitters have a long-range LoRa modem, which can provide long-distance communications (especially in the open field) and most importantly have a high immunity to possible interference that may occur especially in environments working indoors or in cities in general, all these goals are achieved with low energy consumption.

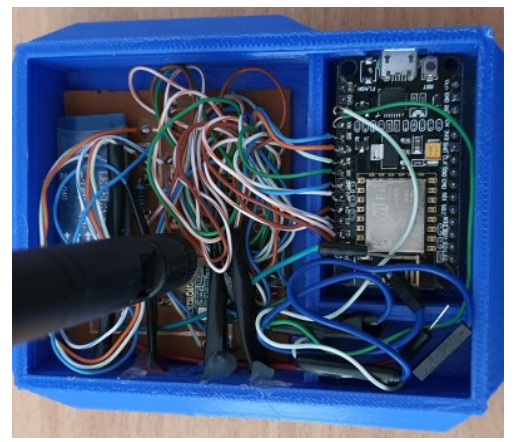

Fig. 5. Presentation of the constructive mode of LoRa nodes 
Using the specific LoRa modulation technique, the RFM96W module has a sensitivity of over $-148 \mathrm{dBm}$. The high sensitivity combined with the integrated power amplifier +20 $\mathrm{dBm}$ produces state-of-the-art equipment in the industry, making it optimal for any application that requires a wide range or robustness. LoRa also offers significant advantages in both blocking and selectivity of conventional modulation techniques, resolving the traditional design trade-off between range, interference immunity and power consumption.

\section{Experimental results}

In order to make an autonomous electric vehicle, we need more data regarding its control method as well as data on the environment and on its location in a given reference space. The first and most important of the tasks is to accurately determine the position of the vehicle in real time in an interior space. Due to the fact that in an interior space and especially in an interior space that has a resistance structure that has strong metal reinforcements, a classical GPS-based location method cannot be used, it is necessary to use another method to determine the position of the vehicle in the reference space with a special accuracy. Thus, taking into account these aspects to determine the position of the autonomous vehicle inside a space, the principle of determining the distance using RSSI (signal strength of the received signal) was used in a network of LoRa devices using the LoRaWAN communication protocol. One of the LoRa devices created was mounted on the vehicle while the other three were mounted on three fixed points. In the first stage, were performed RSSI measurements for all three LoRa devices compared to a fixed LoRA device, the measurements were made every three meters. Starting from the Friis transmission equations and then converting the power from Watt to $\mathrm{dBm}$, a relationship can be obtained between RSSI and distance. RSSI value, simplified here for the reference distance of $1 \mathrm{~m}$, is usually expressed as [17]:

$$
R S S I=-\left(10 \cdot n \cdot \log _{10} d-A\right)
$$

where $\mathrm{A}$ is the power measured in $\mathrm{dBm}$ when the distance between the antenna of the LoRa transmitter (end-node) and the antenna of the LoRa receiver (gateway) is $1 \mathrm{~m}$ (measured experimentally) and $\mathrm{n}$ is the loss factor. This loss factor differs depending on the environment in which the experiments take place and must be measured or calculated for each environment. The distance $\mathrm{d}$ is obtained as [17]:

$$
d=10^{\left(\frac{A-R S S I}{10 n}\right)}
$$

Parameter A is related to the constructive properties of the radio device, while the value of $n$ depends largely on the environment and the operating frequency. The values of $\mathrm{A}$ and $\mathrm{n}$ must be found empirically by measurements.

The algorithm for determining the distance according to the RSSI level was applied to a data set of 12000 values. These data were taken for each of the 3 LoRa nodes in a closed space of the University of Petroșani, a corridor with a length of 81 meters on the 1st floor of the building. The three LoRa modules were arranged in a straight line with a distance between them of 2 meters. Measurements were made every 3 meters and then to obtain the RSSI value specific to a certain point, an arithmetic mean was made of the values taken for each node and for each distance from the central device.

Figure 6 shows the dependency graph of the RSSI indicator as a function of distance for LoRa communication nodes 1, 2 and 3, used for the indoor location and positioning system. 


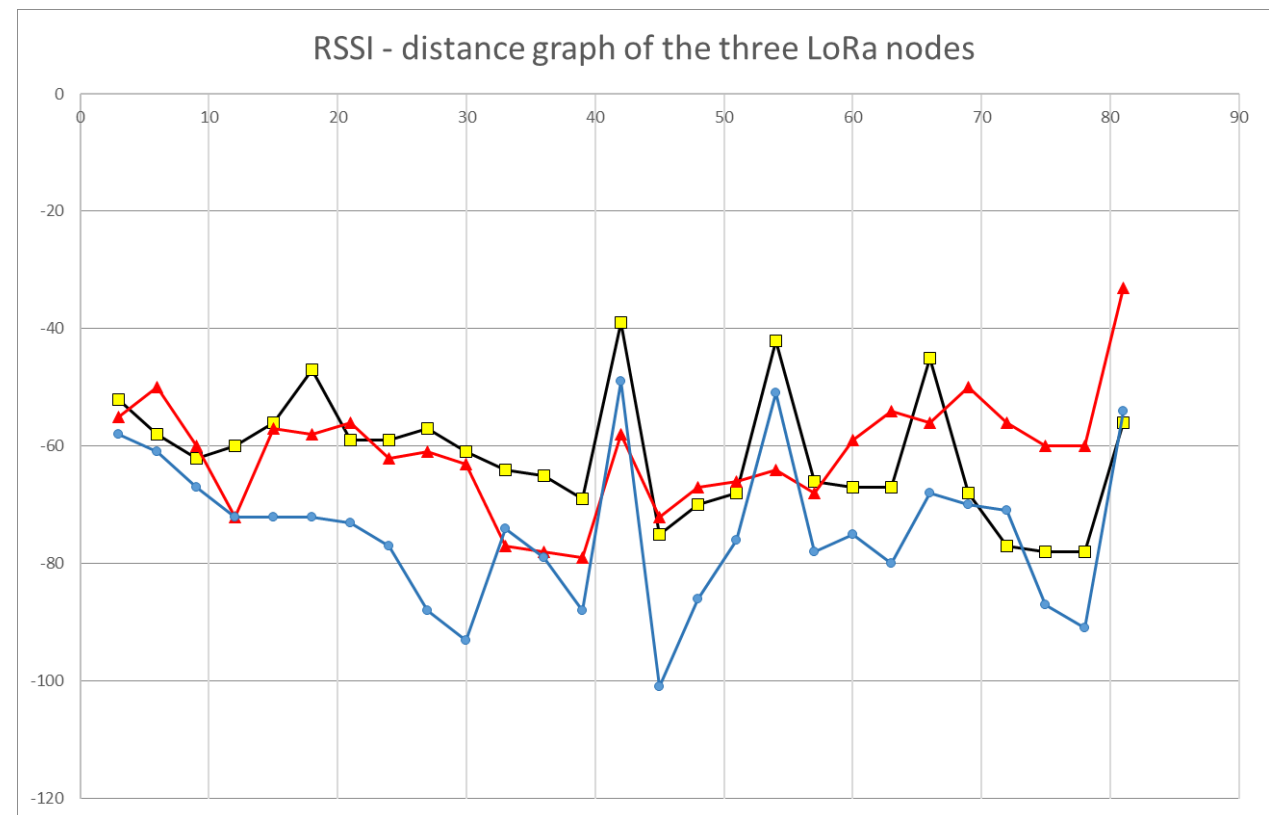

Fig. 6. RSSI-distance graph of the three LoRa nodes

Applying the calculation formula that determines the distance from a certain point of the LoRa network, satisfactory results were obtained only for a distance of up to 21 meters in the conditions of an interior space.

Thus, running the formula for each node of the network it was possible to determine with relative precision its position up to the point of 21 meters from the central device. The measurements regarding the dependence of the RSSI on the distance in a restricted interior space were redone and the following satisfactory results were obtained.

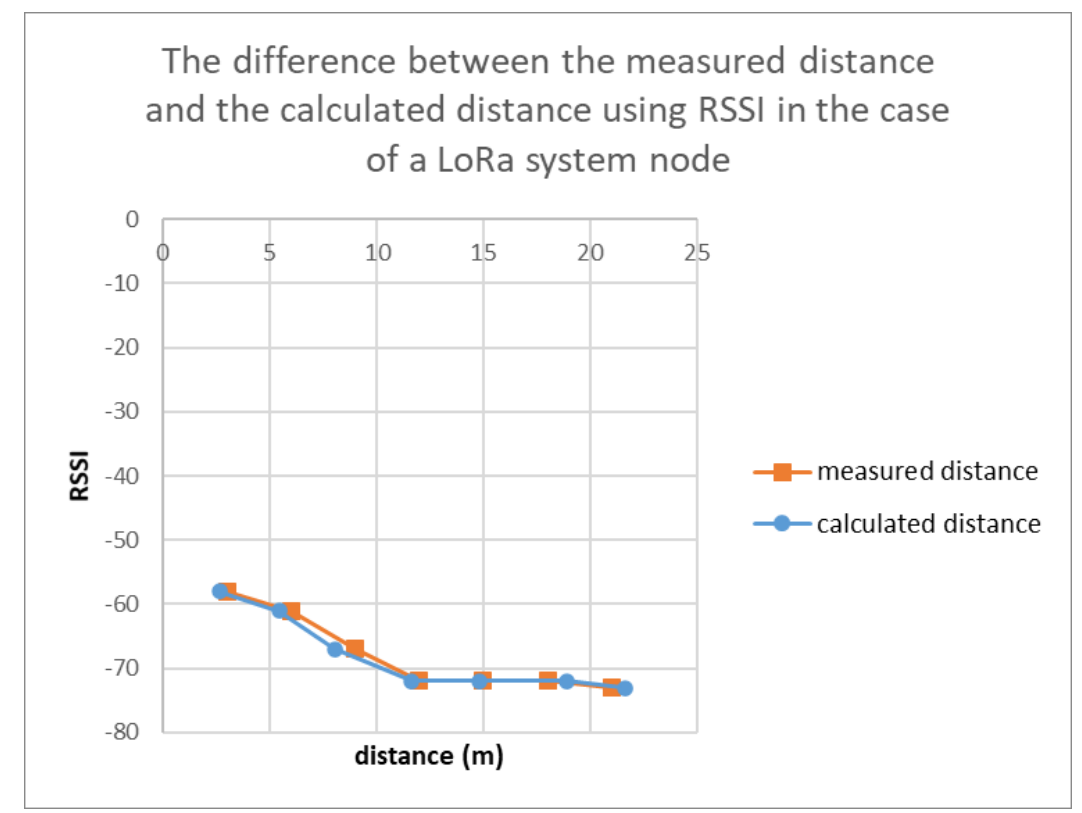

Fig. 7. The graph distance measured - calculated distance 
The graph in figure 7 shows the correlation between the distance of a communication node from a central device obtained by experimental measurements based on the value of the RSSI signal and the value of the distance calculated using the distance calculation algorithm starting from the value of the RSSI signal level (3). It is observed that the two distance curves calculated according to RSSI and the empirically measured distance curve are relatively close, from which we can conclude that the algorithm used is correct although it can be used in specific environmental conditions at the institution only up to 21 meters away. After the threshold of 21 meters, the results obtained are chaotic. This result is also in agreement with the results presented in figure 5 where the major discrepancies between the RSSI signal levels and the distance measured for each of the 3 LoRa nodes are observed, with all these obtained results being satisfactory for this stage of the research.

Table 1 shows the errors in determining the distance using equation (3) from the value of the distance obtained by empirical measurements.

Table 1. Measured distance error - calculated distance

\begin{tabular}{|r|r|r|}
\hline Measured distance (meters) & Calculated distance (meters) & Error \\
\hline 3 & 2,62341352 & $12,6 \%$ \\
\hline 6 & 5,443282347 & $9,3 \%$ \\
\hline 9 & 8,048931925 & $10,6 \%$ \\
\hline 12 & 11,64382931 & $3,0 \%$ \\
\hline 15 & 14,83558551 & $1,1 \%$ \\
\hline 18 & 18,88382931 & $4,9 \%$ \\
\hline 21 & 21,6227766 & $3,0 \%$ \\
\hline
\end{tabular}

It is observed that the error rate is around $10 \%-13 \%$ for short distances while for longer distances of the transmitter from the receiver they start to decrease. This behaviour is related to the resistance structure of the building and to the construction materials used where the tests were performed (1st floor of the University of Petrosani building, building $\mathrm{A}+\mathrm{B})$ and to the possible influences of other equipment that may interfere with LoRa modules. Although it cannot be used on a large scale, the algorithm for determining the distance of an electric vehicle from a reference object using the RSSI value of the LoRa signal, shows good results in laboratory tests and is particularly suitable especially in research to create small equipment to help especially now in the fight against the pandemic of Covid-19 (mobile disinfection devices). Even if the position of an electric vehicle inside a space is known, it is not possible to determine a viable route of it that can be followed in the autonomous regime unless a mapping of the entire space is made in order to observe all possible fixed or mobile obstacles that may occur. Thus, it is necessary to use dedicated tools and algorithms that can generate a map after which a vehicle can run in complete safety. Next, the integration in the Robotic Operating System (ROS) programming environment of the LiDAR sensor is presented and the creation of a map and a possible route that can be followed by the autonomous electric vehicle is highlighted.

Even if the name may give the impression that it is an operating system, in reality, ROS is a framework that runs on Linux or Windows and for MacOS there is an experimental version. In this case, two Raspberry Pi development boards were used, a variant 3 and a variant 4 $\mathrm{B}+$ (thus benefiting from a greater fluency in processing due to the much larger RAM capacity). The ROS installation on the Raspberry Pi was done after an Ubuntu distribution specially created for ARM devices was installed.

Figure 8 shows in graphical form the entire algorithm of operation of the ROS framework used. The whole operating principle is based on ROS core and on different messages between all subsystems [18]. 


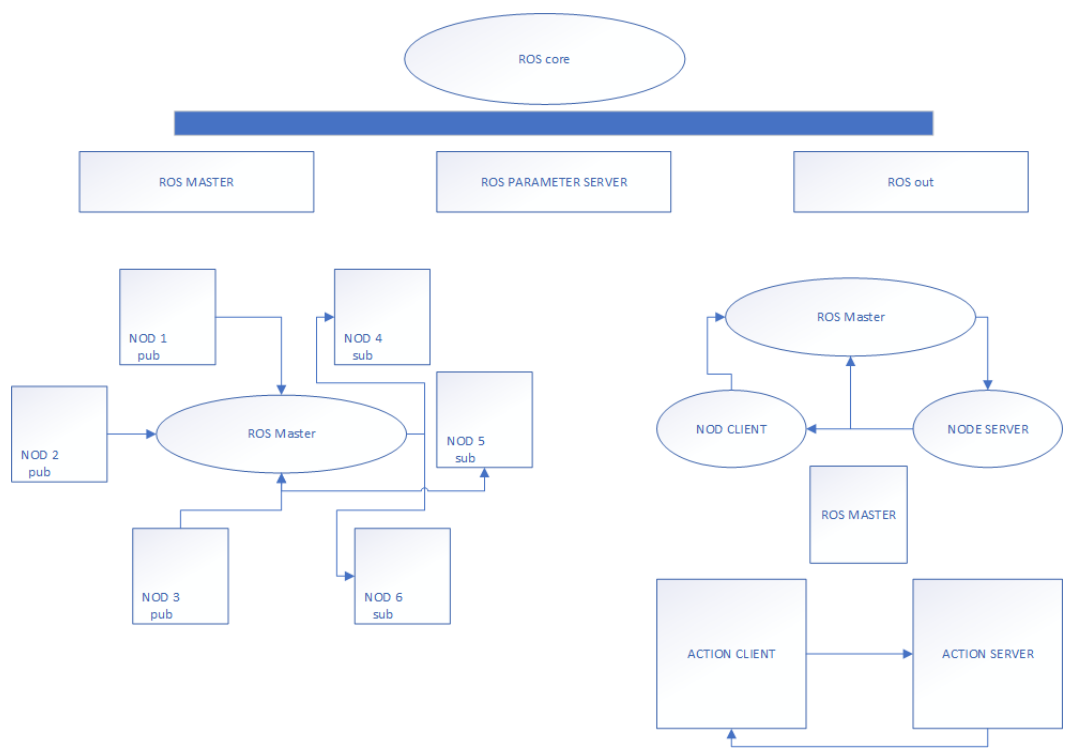

Fig. 8. ROS graphic concept [18]

As shown above, even if the position of the vehicle is known, this is not enough to be able to achieve an autonomous driving algorithm $[19,20]$. At this point the ROS application intervenes. With its help, after the LiDAR RPLiDAR A2 sensor has been integrated, a map of a location can be created inside which the vehicle can move [21].

After all the necessary elements were installed (Ubuntu operating system on a Raspberry Pi 3, video camera, LiDAR A2 sensor) and were integrated as component parts in the ROS application, the hector_slam library was used to make the map of the space in which there were performed the tests [22]. The hector_slam library uses the hector_mapping node to map the environment and simultaneously estimate the $2 \mathrm{D}$ position of the autonomous vehicle at the frame rate of the LiDAR laser scanner [23] (figure 9).

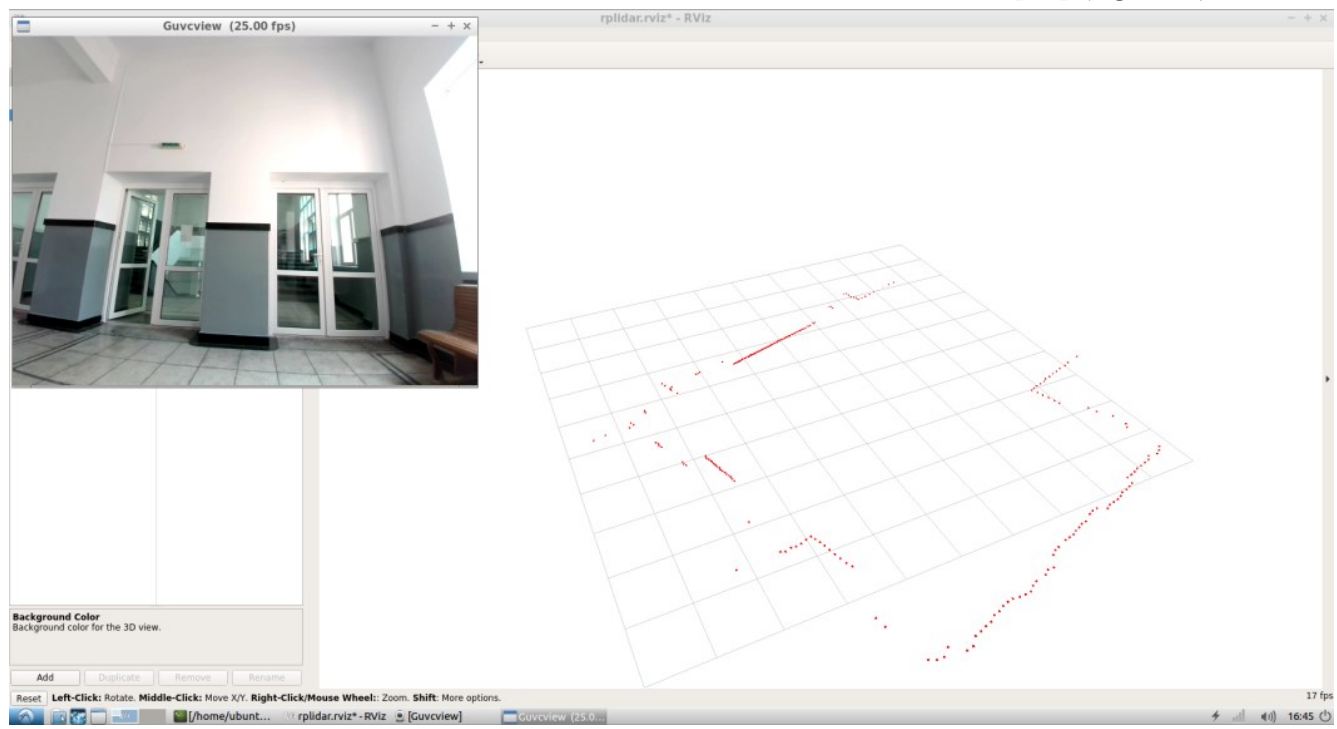

Fig. 9. The correlation between the image of the studied environment and the mapping using RPLiDAR 
Figure 10 shows the notification of an obstacle (in this case, a person) by the LiDAR sensor.

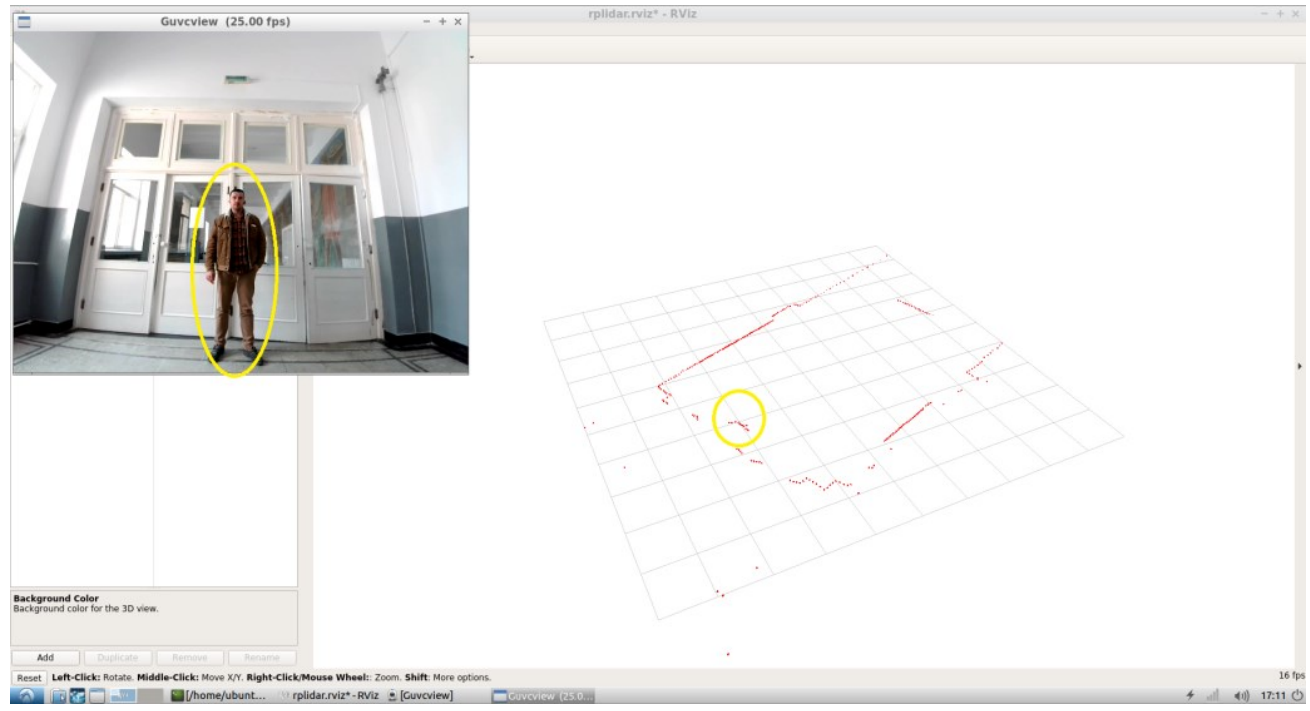

Fig. 10. Representation of detecting a person using RPLiDAR

Figure 11 shows the electric ATV with all the components related to the autonomous navigation system. Within the institution of the University of Petrosani on the first floor of the main building, all the experimental tests were performed. An 81-meter-long corridor was chosen. For the experiments related to the positioning of the vehicle with the help of LoRa and for the experiments related to the drawing of a map of the interior, directed lineof-sight (LOS) configuration was used.

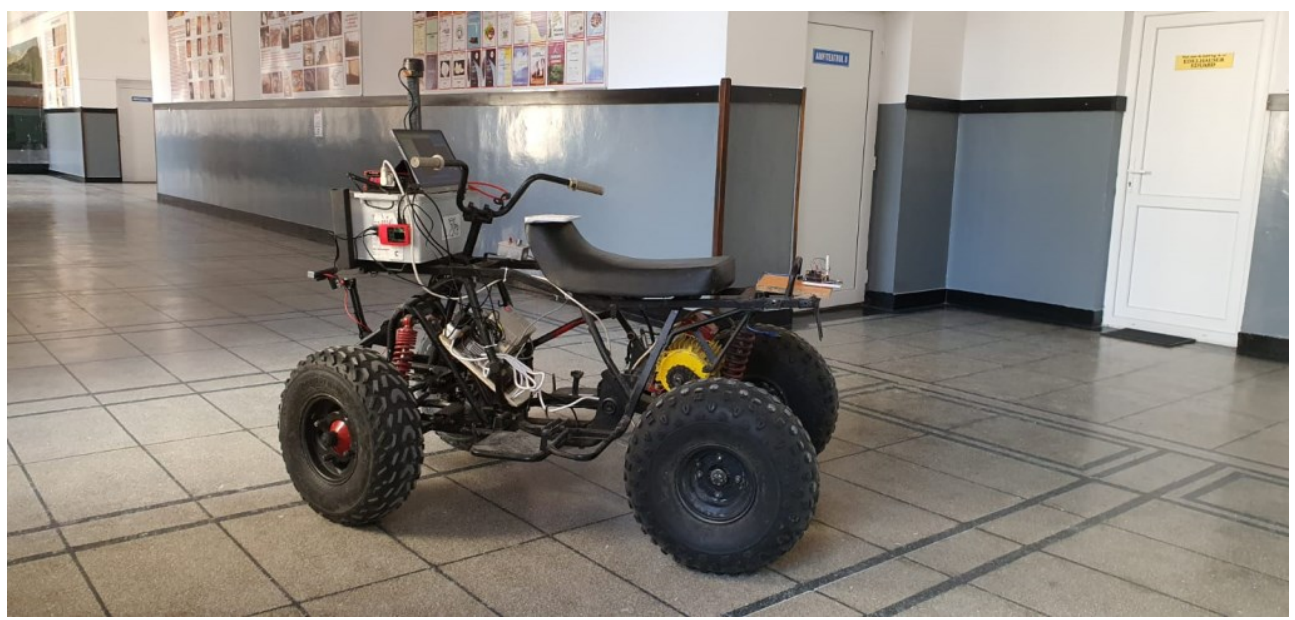

Fig. 11. The electric ATV that has installed the components related to autonomous navigation

The electric vehicle is perfectly functional and is able to transport equipment and people with a payload of approximately 500 kilograms. The steering and braking systems are fully functional and have a high degree of operational reliability. Figure 12 shows the electric ATV that is controlled by a human operator. 


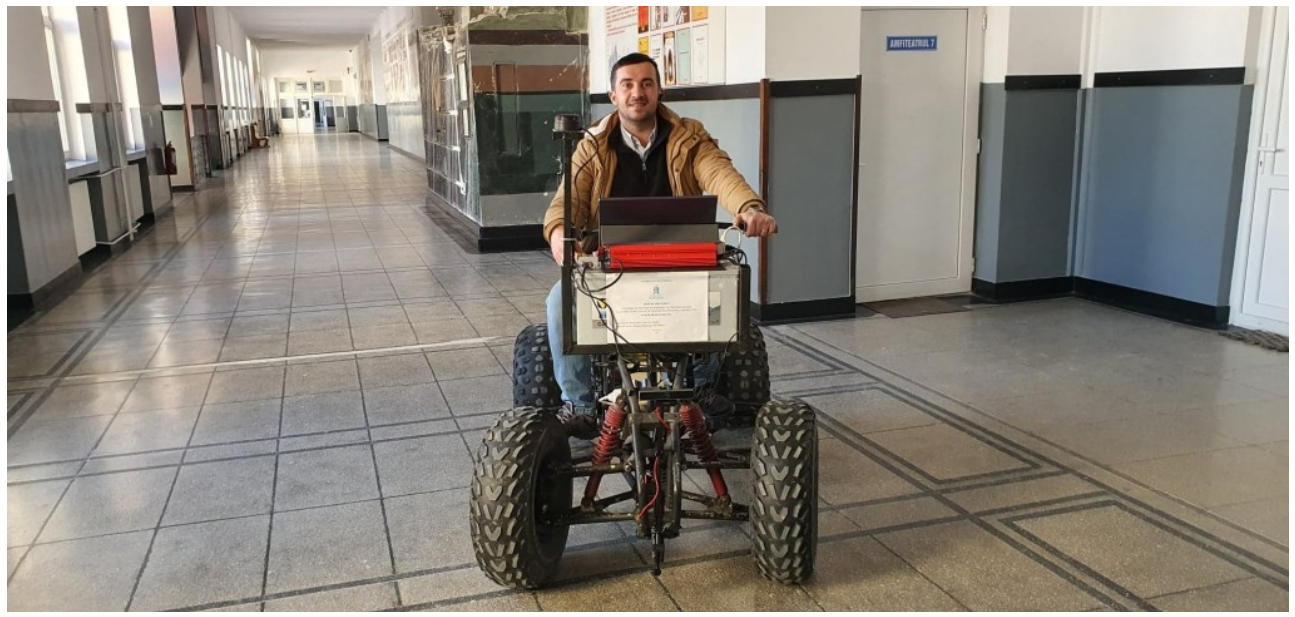

Fig. 12. The electric ATV driven by a human operator

The human operator has at his disposal a touch screen display with which he can observe or control the subsystems located on the functional platform of the vehicle, figure 13. In manual control mode, it is necessary the presence of the human operator who can change the direction of travel, the speed of movement as well as can observe through the touch screen display the system parameters. By including a video camera in the functional assembly of the electric vehicle, a level of video control additional to that of the human operator can be obtained.

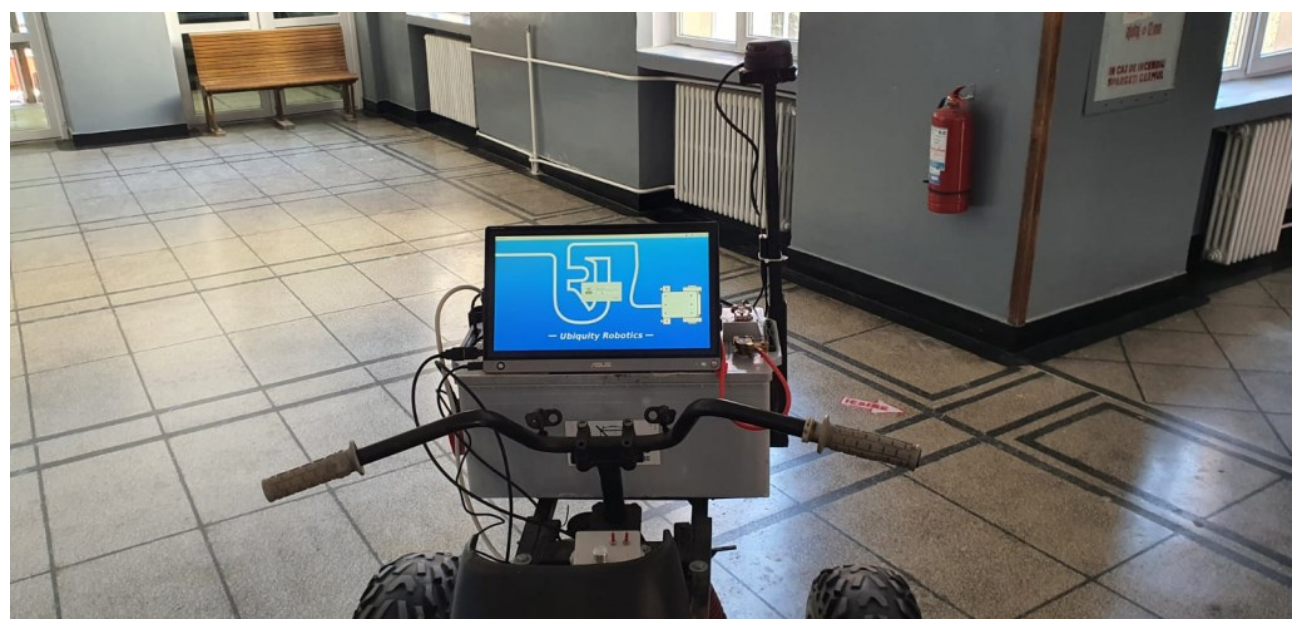

Fig. 13. Electric ATV command and control panel

After the installation, integration and configuration at optimal parameters of the mapping system consisting of the ROS programming environment and the LiDAR sensor on a Raspberry PI development board, the map of the entire corridor of the University of Petrosani in length of 81 meters was made. Particular attention was paid to the correct identification of the obstacles in the studied perimeter. Figure 14 shows the correlation between the position of the electric vehicle using a video camera and the position on the 
map generated with the help of RPLiDAR and a special accuracy is observed in terms of noticing and marking any obstacles that may occur in the way of the vehicle.

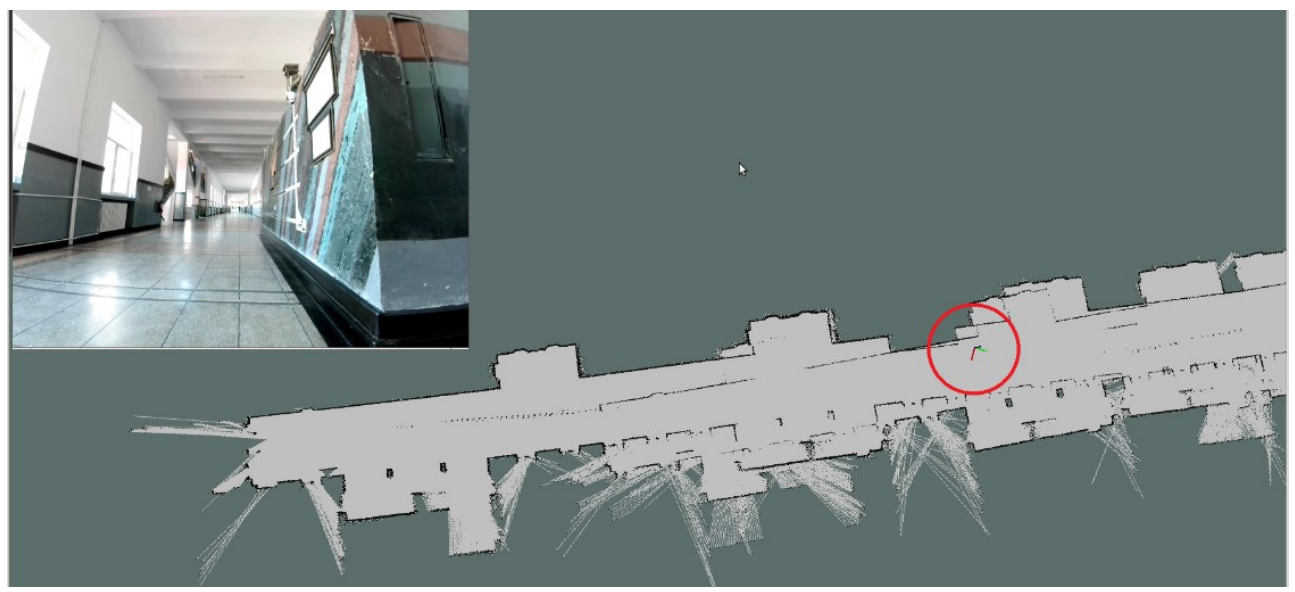

Fig. 14. Notifying and marking an obstacle with the help of RPLiDAR

After the electric vehicle equipped with the RPLiDAR sensor ran three times on the entire surface of the test corridor, it was possible to generate a map that presents a good fidelity in terms of determining and marking all the obstacles encountered. It can be observed both in figure 14 and in figure 15 the fact that all the constructive elements of the studied interior space are clearly represented and each obstacle is marked in full correlation with its real positioning.

After mapping the space, it was possible to draw in real time the route followed by the vehicle while it was moving. The red marking in figure 15 represents the electric vehicle at the starting position.

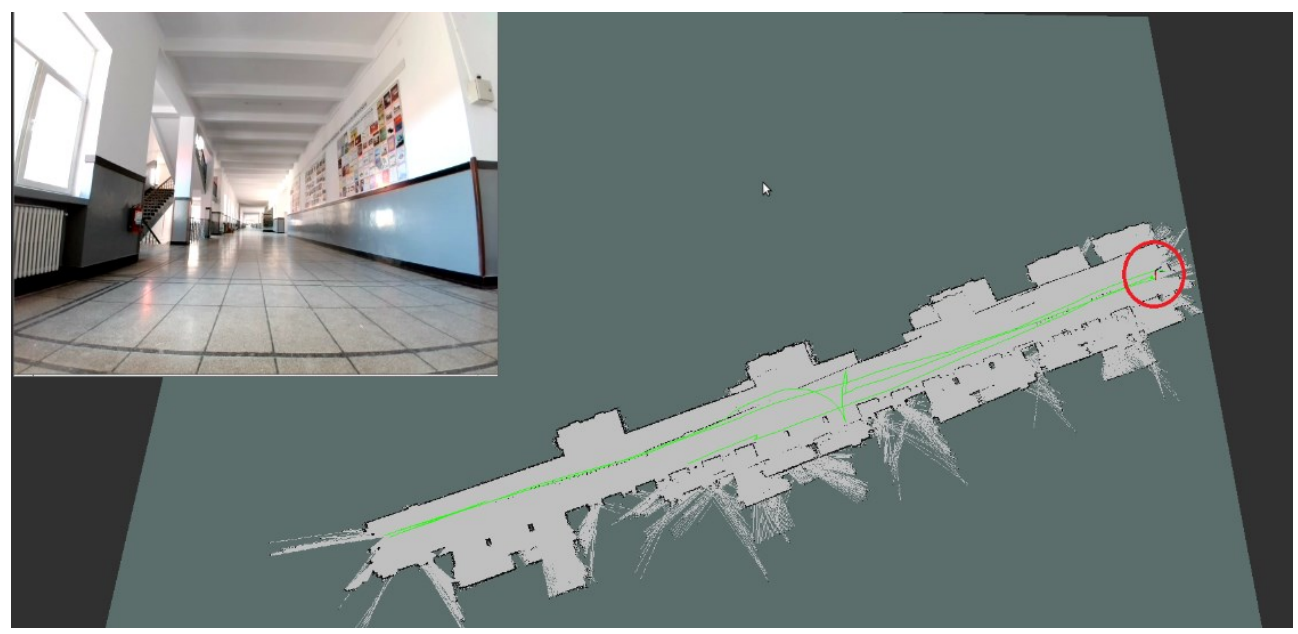

Fig. 15. Generating the route made by the electric vehicle on the map

In order to be fully operational and in terms of autonomous driving, the electric vehicle built so far must also be fitted with a motor that automatically controls the direction 
of movement (left-right) and the possibility of automatic movement selection forward or backward. At the input of the motor driver used for motor control that will allow the automatic selection of the direction of travel (left-right) will be used the data obtained from the integration of the 3 systems presented: LoRa, ROS and RPLiDAR. As further developments, it is desired to use machine learning algorithms and the integration of the vehicle thus created within the concepts of Smart City and Smart Grid in Petrosani [24, 25] against the background of greater interest for autonomous electric vehicles [26].

\section{Conclusions}

The development of an autonomous electric vehicle that meets all the conditions of reliability and specially to ensure a special safety for other traffic participants is a goal that no longer seems so difficult to achieve. All the research carried out so far highlights the benefits that autonomous vehicles can bring, although many questions can be asked, especially of an ethical nature in what an autonomous vehicle can do and what it is allowed to do. This paper laid the foundations for the development of an autonomous electric vehicle starting from the conversion of an ATV type vehicle with internal combustion engine. After the functional construction of the electric vehicle itself was performed, methods were sought to develop a set of algorithms necessary to achieve the desired goal: the autonomous vehicle. First, a vehicle location and positioning system was developed using a LoRa system. One LoRa device was mounted on the functional platform of the vehicle and another three were arranged using a line-of-sight (LOS) configuration at selected points within the entire space ( 81 meters long) of the first-floor corridor of the University of Petroşani. It was thus possible to achieve a positioning of the vehicle in relation to certain fixed points (LoRa beacons) even if as shown in the paper it was possible to obtain an accurate location only in a confined space (part of the entire surface), but for on the whole, it was not enough to know only these data to realize an autonomous driving algorithm. To be able to make a map of the entire test objective (university corridor) and to determine the correct and real-time position of any fixed or mobile obstacles, a hardware and software system consisting of Raspberry Pi, a LiDAR sensor and ROS programming environment together with some specific libraries have been integrated and used. Thus, it was possible to make a map of the entire space on which the electric vehicle can circulate and then it was possible to determine an optimal route. Thus, starting from the realization of a fully functional ATV type electric vehicle, an indoor location system based on LoRa network using the LoRaWAN communication protocol, as well as the inclusion of a system based on LiDAR sensor, it can be said that all these achievements lay the foundations of a safe and reliable autonomous vehicle. All data obtained will serve as input for the components that ensure the effective process of autonomous driving. This vehicle was built based on the current need to provide disinfected interior spaces in the current pandemic context. Thus, this autonomous vehicle will also have a surface disinfection system made with the help of ultraviolet lamps and will thus be able to disinfect the common spaces within the University of Petrosani to combat the COVID-19 epidemic (SARS-COV2). The autonomous driving component will allow the vehicle to travel on a well-defined route without the need for the presence of a human operator who in the conditions of a classic vehicle would have been exposed to the entire disinfection process, UV-C ultraviolet lamps neutralize viruses from Sars-Cov spectrum but are dangerous to health in the event of direct exposure.

This paper has achieved its purpose and lays the foundations for the development of autonomous electric vehicles within the University of Petroșani. 


\section{References}

1. S.A Bagloee, M. Tavana, M. Asadi, T. Oliver, J. Mod. Transp., 24(4), 284-303 (2016)

2. M. J. Anderson, N. Kalra, K. D. Stanley, P. Sorensen, C. Samaras, T. A. Oluwatola, Autonomous Vehicle Technology: A Guide for Policymakers (RAND Corporation, Santa Monica, Calif., 2016)

3. S. Saponara, M. S. Greco, F. Gini, IEEE Signal Process. Mag., 36(5), 71-84 (2019)

4. X. Ma, X. Hu, S. Schweig, J. Pragalathan, D. Schramm, Appl. Sci., 11(1), 380 (2021)

5. S.M. Mousavi, O.A. Osman, D. Lord, K.K. Dixon, B. Dadashova, Accid. Anal. Prev., $152(2012)$

6. S. Koos, Yuridika, 36(1), 235-262 (2021)

7. S. Riurean, M. Leba, A. Ionica, Indoor positioning and guiding system based on VLC for visually impaired people, The 2019 International Conference on Digital Science, DSIC'19, Limassol, Cyprus, (2019)

8. S. Rosca, S. Riurean, M. Leba, A. Ionica, A reliable wireless communication system for hazardous environments, The 2018 Intenational conference on Digital Science DSIC'18, Budva, Montenegro, (2018)

9. S. Liu, IOP Conf. Ser.: Earth Environ. Sci. 692022103 (2021)

10. C. Rus, N. Mija, M. Leba, Autonomous electric ATV using IPM based inverter control and deep learning, World Conference on Information Systems and Technologies, WorldCIST'20, 746-755, Budva, Montenegro, (2020)

11. C. Rus, R. Marcus, L. Pellegrini, M. Leba, M. Rebrisoreanu, A. Constandoiu, IOP Conf. Ser.: Mater. Sci. Eng. 572012091 (2019)

12. X. Chen, I. Vizzo, T. Läbe, J. Behley, C. Stachniss, Range Image-based LiDAR localization for autonomous vehicles, ICRA 2021, Xi'an, China, (2021)

13. G. Wang, J. Wu, T. Xu, B. Tian, IEEE Trans. Veh. Technol., 70(1), 344-355 (2021)

14. G. Spampinato, A. Bruna, I. Guarneri, D. Giacalone, , Deep learning localization with 2 D range scanner, ICARA 2021, Prague, Czech Republic, (2021)

15. C. Rus, M. Leba, R. Marcuş, L. Pellegrini, A. Costandoiu, LoRa communication and geolocation system for sensors network SESAM 2019, Petrosani, Romania, (2019)

16. C. Rus, N. Negru, P. Patrascoiu, J. Environ. Prot. Ecol., 20 (3), 1451-1461 (2019)

17. E. Goldoni, L. Prando, A. Vizziello, P. Savazzi, P. Gamba, Internet Technol. Lett., 2, 75 (2019)

18. H. Deng, Z. Xia, S. Weng, Y. Gan, P. Fang, J. Xiong, Robot. Biomim. 3 (2016)

19. A. Lorincz, M. N. Risteiu, A. Constandoiu, Development of a drive management system by V2I communication, MSE 2017, Sibiu, Romania, (2017)

20. A. Lorincz, M.-N. Risteiu, M. Leba, A. Ionica, , Driver monitoring system for automotive safety, ICAS 2017, Hunedoara, Romania, (2017)

21. S. Grigorescu, B. Trasnea, T. Cocias, G. Macesanu, J. Field Robot., 37(3), 362-386 (2020)

22. H. Alaa Aldeen, G. Xingyu, , Simultaneous Localization and Mapping using differential drive mobile robot under ROS, MEMAT 2021, Guilin, China, (2021)

23. S. G. Bogdanov, D. S. Chikurtev, N. R. Spasova, ,Embedded system environment selfawareness using LIDAR technologies for robotics applications, ICTTE 2020, Yambol, Bulgaria, (2020)

24. M. Rebrisoreanu, C. Rus, M. Leba, A. Ionica, Int. J. Syst. Appl. Eng. Dev., 12 164-167 (2018)

25. R. Marcuş, O. Stoicuţa, C. Rus, B. Tomus, Exploring the possibilities to increase the autonomy of an electric vehicle, SESAM 2019, Petrosani, Romania, (2019)

26. https://www2.deloitte.com/uk/en/insights/focus/future-of-mobility/electric-vehicletrends-2030.html, [Accessed 12.02.2021] 